Internat. J. Math. \& Math. Sci.

Vol. 24, No. 12 (2000) 839-849

S0161171200004610

(C) Hindawi Publishing Corp.

\title{
INTUITIONISTIC FUZZY IDEALS OF BCK-ALGEBRAS
}

\author{
YOUNG BAE JUN and KYUNG HO KIM
}

(Received 16 February 2000)

\begin{abstract}
We consider the intuitionistic fuzzification of the concept of subalgebras and ideals in BCK-algebras, and investigate some of their properties. We introduce the notion of equivalence relations on the family of all intuitionistic fuzzy ideals of a BCK-algebra and investigate some related properties.
\end{abstract}

Keywords and phrases. (Intuitionistic) fuzzy subalgebra, (intuitionistic) fuzzy ideal, upper (respectively, lower) $t$-level cut, homomorphism.

2000 Mathematics Subject Classification. Primary 06F35, 03G25, 03E72.

1. Introduction. After the introduction of the concept of fuzzy sets by Zadeh [9] several researches were conducted on the generalizations of the notion of fuzzy sets. The idea of "intuitionistic fuzzy set" was first published by Atanassov [1, 2], as a generalization of the notion of fuzzy set. The first author (together with Hong, Kim, Kim, Meng, Roh, and Song) considered the fuzzification of ideals and subalgebras in BCK-algebras (cf. [3, 4, 5, 6, 7, 8]). In this paper, using the Atanassov's idea, we establish the intuitionistic fuzzification of the concept of subalgebras and ideals in BCK-algebras, and investigate some of their properties. We introduce the notion of equivalence relations on the family of all intuitionistic fuzzy ideals of a BCK-algebra and investigate some related properties.

2. Preliminaries. First we present the fundamental definitions. By a BCK-algebra we mean a nonempty set $X$ with a binary operation $*$ and a constant 0 satisfying the following conditions:

(I) $((x * y) *(x * z)) *(z * y)=0$,

(II) $(x *(x * y)) * y=0$,

(III) $x * x=0$,

(IV) $0 * x=0$,

(V) $x * y=0$ and $y * x=0$ imply that $x=y$

for all $x, y, z \in X$.

A partial ordering " $\leq$ " on $X$ can be defined by $x \leq y$ if and only if $x * y=0$. A nonempty subset $S$ of a BCK-algebra $X$ is called a subalgebra of $X$ if $x * y \in S$ whenever $x, y \in S$. A nonempty subset $I$ of a BCK-algebra $X$ is called an ideal of $X$ if

(i) $0 \in I$,

(ii) $x * y \in I$ and $y \in I$ imply that $x \in I$ for all $x, y \in X$.

By a fuzzy set $\mu$ in a nonempty set $X$ we mean a function $\mu: X \rightarrow[0,1]$, and the complement of $\mu$, denoted by $\bar{\mu}$, is the fuzzy set in $X$ given by $\bar{\mu}(x)=1-\mu(x)$ for all $x \in X$. A fuzzy set $\mu$ in a BCK-algebra $X$ is called a fuzzy subalgebra of $X$ if $\mu(x * y) \geq$ 
$\min \{\mu(x), \mu(y)\}$ for all $x, y \in X$. A fuzzy set $\mu$ in a BCK-algebra $X$ is called a fuzzy ideal of $X$ if

(i) $\mu(0) \geq \mu(x)$ for all $x \in X$,

(ii) $\mu(x) \geq \min \{\mu(x * y), \mu(y)\}$ for all $x, y \in X$.

An intuitionistic fuzzy set (briefly, IFS) $A$ in a nonempty set $X$ is an object having the form

$$
A=\left\{\left(x, \alpha_{A}(x), \beta_{A}(x)\right) \mid x \in X\right\},
$$

where the functions $\alpha_{A}: X \rightarrow[0,1]$ and $\beta_{A}: X \rightarrow[0,1]$ denote the degree of membership and the degree of nonmembership, respectively, and

$$
0 \leq \alpha_{A}(x)+\beta_{A}(x) \leq 1 \quad \forall x \in X .
$$

An intuitionistic fuzzy set $A=\left\{\left(x, \alpha_{A}(x), \beta_{A}(x)\right) \mid x \in X\right\}$ in $X$ can be identified to an ordered pair $\left(\alpha_{A}, \beta_{A}\right)$ in $I^{X} \times I^{X}$. For the sake of simplicity, we shall use the symbol $A=\left(\alpha_{A}, \beta_{A}\right)$ for the IFS $A=\left\{\left(x, \alpha_{A}(x), \beta_{A}(x)\right) \mid x \in X\right\}$.

3. Intuitionistic fuzzy ideals. In what follows, let $X$ denote a BCK-algebra unless otherwise specified.

DeFINITION 3.1. An IFS $A=\left(\alpha_{A}, \beta_{A}\right)$ in $X$ is called an intuitionistic fuzzy subalgebra of $X$ if it satisfies:

(IS1) $\alpha_{A}(x * y) \geq \min \left\{\alpha_{A}(x), \alpha_{A}(y)\right\}$,

(IS2) $\beta_{A}(x * y) \leq \max \left\{\beta_{A}(x), \beta_{A}(y)\right\}$,

for all $x, y \in X$.

EXAMPLE 3.2. Consider a BCK-algebra $X=\{0, a, b, c\}$ with the following Cayley table:

\begin{tabular}{l|llll}
\hline$*$ & 0 & $a$ & $b$ & $c$ \\
\hline 0 & 0 & 0 & 0 & 0 \\
$a$ & $a$ & 0 & 0 & $a$ \\
$b$ & $b$ & $a$ & 0 & $b$ \\
$c$ & $c$ & $c$ & $c$ & 0 \\
\hline
\end{tabular}

Let $A=\left(\alpha_{A}, \beta_{A}\right)$ be an IFS in $X$ defined by

$$
\begin{aligned}
& \alpha_{A}(0)=\alpha_{A}(a)=\alpha_{A}(c)=0.7>0.3=\alpha_{A}(b), \\
& \beta_{A}(0)=\beta_{A}(a)=\beta_{A}(c)=0.2<0.5=\beta_{A}(b) .
\end{aligned}
$$

Then $A=\left(\alpha_{A}, \beta_{A}\right)$ is an intuitionistic fuzzy subalgebra of $X$.

Proposition 3.3. Every intuitionistic fuzzy subalgebra $A=\left(\alpha_{A}, \beta_{A}\right)$ of $X$ satisfies the inequalities $\alpha_{A}(0) \geq \alpha_{A}(x)$ and $\beta_{A}(0) \leq \beta_{A}(x)$ for all $x \in X$.

Proof. For any $x \in X$, we have

$$
\begin{aligned}
& \alpha_{A}(0)=\alpha_{A}(x * x) \geq \min \left\{\alpha_{A}(x), \alpha_{A}(x)\right\}=\alpha_{A}(x), \\
& \beta_{A}(0)=\beta_{A}(x * x) \leq \max \left\{\beta_{A}(x), \beta_{A}(x)\right\}=\beta_{A}(x) .
\end{aligned}
$$

This completes the proof. 
DEFINITION 3.4. An IFS $A=\left(\alpha_{A}, \beta_{A}\right)$ in $X$ is called an intuitionistic fuzzy ideal of $X$ if it satisfies the following inequalities:

(IF1) $\alpha_{A}(0) \geq \alpha_{A}(x)$ and $\beta_{A}(0) \leq \beta_{A}(x)$,

(IF2) $\alpha_{A}(x) \geq \min \left\{\alpha_{A}(x * y), \alpha_{A}(y)\right\}$,

(IF3) $\beta_{A}(x) \leq \max \left\{\beta_{A}(x * y), \beta_{A}(y)\right\}$,

for all $x, y \in X$.

EXAMPLE 3.5. Let $X=\{0,1,2,3,4\}$ be a BCK-algebra with the following Cayley table:

\begin{tabular}{l|lllll}
\hline$*$ & 0 & 1 & 2 & 3 & 4 \\
\hline 0 & 0 & 0 & 0 & 0 & 0 \\
1 & 1 & 0 & 1 & 0 & 0 \\
2 & 2 & 2 & 0 & 0 & 0 \\
3 & 3 & 3 & 3 & 0 & 0 \\
4 & 4 & 3 & 4 & 1 & 0 \\
\hline
\end{tabular}

Define an IFS $A=\left(\alpha_{A}, \beta_{A}\right)$ in $X$ as follows:

$$
\begin{gathered}
\alpha_{A}(0)=\alpha_{A}(2)=1, \quad \alpha_{A}(1)=\alpha_{A}(3)=\alpha_{A}(4)=t, \\
\beta_{A}(0)=\beta_{A}(2)=0, \quad \beta_{A}(1)=\beta_{A}(3)=\beta_{A}(4)=s,
\end{gathered}
$$

where $t \in[0,1], s \in[0,1]$, and $t+s \leq 1$. By routine calculation we know that $A=$ $\left(\alpha_{A}, \beta_{A}\right)$ is an intuitionistic fuzzy ideal of $X$.

LEMMA 3.6. Let an IFS $A=\left(\alpha_{A}, \beta_{A}\right)$ in $X$ be an intuitionistic fuzzy ideal of $X$. If the inequality $x * y \leq z$ holds in $X$, then

$$
\alpha_{A}(x) \geq \min \left\{\alpha_{A}(y), \alpha_{A}(z)\right\}, \quad \beta_{A}(x) \leq \max \left\{\beta_{A}(y), \beta_{A}(z)\right\} .
$$

Proof. Let $x, y, z \in X$ be such that $x * y \leq z$. Then $(x * y) * z=0$, and thus

$$
\begin{aligned}
\alpha_{A}(x) & \geq \min \left\{\alpha_{A}(x * y), \alpha_{A}(y)\right\} \\
& \geq \min \left\{\min \left\{\alpha_{A}((x * y) * z), \alpha_{A}(z)\right\}, \alpha_{A}(y)\right\} \\
& =\min \left\{\min \left\{\alpha_{A}(0), \alpha_{A}(z)\right\}, \alpha_{A}(y)\right\} \\
& =\min \left\{\alpha_{A}(y), \alpha_{A}(z)\right\}, \\
\beta_{A}(x) & \leq \max \left\{\beta_{A}(x * y), \beta_{A}(y)\right\} \\
& \leq \max \left\{\max \left\{\beta_{A}((x * y) * z), \beta_{A}(z)\right\}, \beta_{A}(y)\right\} \\
& =\max \left\{\max \left\{\beta_{A}(0), \beta_{A}(z)\right\}, \beta_{A}(y)\right\} \\
& =\max \left\{\beta_{A}(y), \beta_{A}(z)\right\},
\end{aligned}
$$

this completes the proof.

LEMMA 3.7. Let $A=\left(\alpha_{A}, \beta_{A}\right)$ be an intuitionistic fuzzy ideal of $X$. If $x \leq y$ in $X$, then

$$
\alpha_{A}(x) \geq \alpha_{A}(y), \quad \beta_{A}(x) \leq \beta_{A}(y),
$$

that is, $\alpha_{A}$ is order-reserving and $\beta_{A}$ is order-preserving. 
Proof. Let $x, y \in X$ be such that $x \leq y$. Then $x * y=0$ and so

$$
\begin{aligned}
& \alpha_{A}(x) \geq \min \left\{\alpha_{A}(x * y), \alpha_{A}(y)\right\}=\min \left\{\alpha_{A}(0), \alpha_{A}(y)\right\}=\alpha_{A}(y), \\
& \beta_{A}(x) \leq \max \left\{\beta_{A}(x * y), \beta_{A}(y)\right\}=\max \left\{\beta_{A}(0), \beta_{A}(y)\right\}=\beta_{A}(y) .
\end{aligned}
$$

This completes the proof.

THEOREM 3.8. If $A=\left(\alpha_{A}, \beta_{A}\right)$ is an intuitionistic fuzzy ideal of $X$, then for any $x, a_{1}, a_{2}, \ldots, a_{n} \in X,\left(\cdots\left(\left(x * a_{1}\right) * a_{2}\right) * \cdots\right) * a_{n}=0$ implies

$$
\begin{aligned}
& \alpha_{A}(x) \geq \min \left\{\alpha_{A}\left(a_{1}\right), \alpha_{A}\left(a_{2}\right), \ldots, \alpha_{A}\left(a_{n}\right)\right\}, \\
& \beta_{A}(x) \leq \max \left\{\beta_{A}\left(a_{1}\right), \beta_{A}\left(a_{2}\right), \ldots, \beta_{A}\left(a_{n}\right)\right\} .
\end{aligned}
$$

Proof. Using induction on $n$ and Lemmas 3.6 and 3.7, the proof is straightforward.

THEOREM 3.9. Every intuitionistic fuzzy ideal of $X$ is an intuitionistic fuzzy subalgebra of $X$.

Proof. Let $A=\left(\alpha_{A}, \beta_{A}\right)$ be an intuitionistic fuzzy ideal of $X$. Since $x * y \leq x$ for all $x, y \in X$, it follows from Lemma 3.7 that

$$
\alpha_{A}(x * y) \geq \alpha_{A}(x), \quad \beta_{A}(x * y) \leq \beta_{A}(x),
$$

so by (IF2) and (IF3),

$$
\begin{aligned}
& \alpha_{A}(x * y) \geq \alpha_{A}(x) \geq \min \left\{\alpha_{A}(x * y), \alpha_{A}(y)\right\} \geq \min \left\{\alpha_{A}(x), \alpha_{A}(y)\right\}, \\
& \beta_{A}(x * y) \leq \beta_{A}(x) \leq \max \left\{\beta_{A}(x * y), \beta_{A}(y)\right\} \leq \max \left\{\beta_{A}(x), \beta_{A}(y)\right\} .
\end{aligned}
$$

This shows that $A=\left(\alpha_{A}, \beta_{A}\right)$ is an intuitionistic fuzzy subalgebra of $X$.

The converse of Theorem 3.9 may not be true. For example, the intuitionistic fuzzy subalgebra $A=\left(\alpha_{A}, \beta_{A}\right)$ in Example 3.2 is not an intuitionistic fuzzy ideal of $X$ since

$$
\beta_{A}(b)=0.5>0.2=\min \left\{\beta_{A}(b * a), \beta_{A}(a)\right\} .
$$

We now give a condition for an intuitionistic fuzzy subalgebra to be an intuitionistic fuzzy ideal.

THEOREM 3.10. Let $A=\left(\alpha_{A}, \beta_{A}\right)$ be an intuitionistic fuzzy subalgebra of $X$ such that

$$
\alpha_{A}(x) \geq \min \left\{\alpha_{A}(y), \alpha_{A}(z)\right\}, \quad \beta_{A}(x) \leq \max \left\{\beta_{A}(y), \beta_{A}(z)\right\}
$$

for all $x, y, z \in X$ satisfying the inequality $x * y \leq z$. Then $A=\left(\alpha_{A}, \beta_{A}\right)$ is an intuitionistic fuzzy ideal of $X$.

Proof. Let $A=\left(\alpha_{A}, \beta_{A}\right)$ be an intuitionistic fuzzy subalgebra of $X$. Recall that $\alpha_{A}(0) \geq \alpha_{A}(x)$ and $\beta_{A}(0) \leq \beta_{A}(x)$ for all $X$. Since $x *(x * y) \leq y$, it follows from the hypothesis that

$$
\alpha_{A}(x) \geq \min \left\{\alpha_{A}(x * y), \alpha_{A}(y)\right\}, \quad \beta_{A}(x) \leq \max \left\{\beta_{A}(x * y), \beta_{A}(y)\right\} .
$$

Hence $A=\left(\alpha_{A}, \beta_{A}\right)$ is an intuitionistic fuzzy ideal of $X$. 
LEMMA 3.11. An IFS $A=\left(\alpha_{A}, \beta_{A}\right)$ is an intuitionistic fuzzy ideal of $X$ if and only if the fuzzy sets $\alpha_{A}$ and $\bar{\beta}_{A}$ are fuzzy ideals of $X$.

Proof. Let $A=\left(\alpha_{A}, \beta_{A}\right)$ be an intuitionistic fuzzy ideal of $X$. Clearly, $\alpha_{A}$ is a fuzzy ideal of $X$. For every $x, y \in X$, we have

$$
\begin{aligned}
\bar{\beta}_{A}(0) & =1-\beta_{A}(0) \geq 1-\beta_{A}(x)=\bar{\beta}_{A}(x), \\
\bar{\beta}_{A}(x) & =1-\beta_{A}(x) \geq 1-\max \left\{\beta_{A}(x * y), \beta_{A}(y)\right\} \\
& =\min \left\{1-\beta_{A}(x * y), 1-\beta_{A}(y)\right\} \\
& =\min \left\{\bar{\beta}_{A}(x * y), \bar{\beta}_{A}(y)\right\} .
\end{aligned}
$$

Hence $\bar{\beta}_{A}$ is a fuzzy ideal of $X$.

Conversely, assume that $\alpha_{A}$ and $\bar{\beta}_{A}$ are fuzzy ideals of $X$. For every $x, y \in X$, we get

$$
\alpha_{A}(0) \geq \alpha_{A}(x), \quad 1-\beta_{A}(0)=\bar{\beta}_{A}(0) \geq \bar{\beta}_{A}(x)=1-\beta_{A}(x),
$$

that is, $\beta_{A}(0) \leq \beta_{A}(x) ; \alpha_{A}(x) \geq \min \left\{\alpha_{A}(x * y), \alpha_{A}(y)\right\}$ and

$$
\begin{aligned}
1-\beta_{A}(x) & =\bar{\beta}_{A}(x) \geq \min \left\{\bar{\beta}_{A}(x * y), \bar{\beta}_{A}(y)\right\} \\
& =\min \left\{1-\beta_{A}(x * y), 1-\beta_{A}(y)\right\} \\
& =1-\max \left\{\beta_{A}(x * y), \beta_{A}(y)\right\},
\end{aligned}
$$

that is, $\beta_{A}(x) \leq \max \left\{\beta_{A}(x * y), \beta_{A}(y)\right\}$. Hence $A=\left(\alpha_{A}, \beta_{A}\right)$ is an intuitionistic fuzzy ideal of $X$.

THEOREM 3.12. Let $A=\left(\alpha_{A}, \beta_{A}\right)$ be an IFS in $X$. Then $A=\left(\alpha_{A}, \beta_{A}\right)$ is an intuitionistic fuzzy ideal of $X$ if and only if $\square A=\left(\alpha_{A}, \bar{\alpha}_{A}\right)$ and $\diamond A=\left(\bar{\beta}_{A}, \beta_{A}\right)$ are intuitionistic fuzzy ideals of $X$.

Proof. If $A=\left(\alpha_{A}, \beta_{A}\right)$ is an intuitionistic fuzzy ideal of $X$, then $\alpha_{A}=\overline{\bar{\alpha}}_{A}$ and $\beta_{A}$ are fuzzy ideals of $X$ from Lemma 3.11, hence $\square A=\left(\alpha_{A}, \bar{\alpha}_{A}\right)$ and $\diamond A=\left(\bar{\beta}_{A}, \beta_{A}\right)$ are intuitionistic fuzzy ideals of $X$. Conversely, if $\square A=\left(\alpha_{A}, \bar{\alpha}_{A}\right)$ and $\diamond A=\left(\bar{\beta}_{A}, \beta_{A}\right)$ are intuitionistic fuzzy ideals of $X$, then the fuzzy sets $\alpha_{A}$ and $\bar{\beta}_{A}$ are fuzzy ideals of $X$, hence $A=\left(\alpha_{A}, \beta_{A}\right)$ is an intuitionistic fuzzy ideal of $X$.

For any $t \in[0,1]$ and a fuzzy set $\mu$ in a nonempty set $X$, the set

$$
U(\mu ; t)=\{x \in X \mid \mu(x) \geq t\}
$$

is called an upper $t$-level cut of $\mu$ and the set

$$
L(\mu ; t)=\{x \in X \mid \mu(x) \leq t\}
$$

is called a lower t-level cut of $\mu$.

THEOREM 3.13. An IFS $A=\left(\alpha_{A}, \beta_{A}\right)$ is an intuitionistic fuzzy ideal of $X$ if and only if for all $s, t \in[0,1]$, the sets $U\left(\alpha_{A} ; t\right)$ and $L\left(\beta_{A} ; s\right)$ are either empty or ideals of $X$. 
Proof. Let $A=\left(\alpha_{A}, \beta_{A}\right)$ be an intuitionistic fuzzy ideal of $X$ and $U\left(\alpha_{A} ; t\right) \neq \varnothing \neq$ $L\left(\beta_{A} ; s\right)$ for any $s, t \in[0,1]$. It is clear that $0 \in U\left(\alpha_{A} ; t\right) \cap L\left(\beta_{A} ; s\right)$ since $\alpha_{A}(0) \geq t$ and $\beta_{A}(0) \leq s$. Let $x, y \in X$ be such that $x * y \in U\left(\alpha_{A} ; t\right)$ and $y \in U\left(\alpha_{A} ; t\right)$. Then $\alpha_{A}(x * y) \geq t$ and $\alpha_{A}(y) \geq t$. It follows that

$$
\alpha_{A}(x) \geq \min \left\{\alpha_{A}(x * y), \alpha_{A}(y)\right\} \geq t
$$

so that $x \in U\left(\alpha_{A} ; t\right)$. Hence $U\left(\alpha_{A} ; t\right)$ is an ideal of $X$. Now let $x, y \in X$ be such that $x * y \in L\left(\beta_{A} ; s\right)$ and $y \in L\left(\beta_{A} ; s\right)$. Then $\beta_{A}(x * y) \leq s$ and $\beta_{A}(y) \leq s$, which imply that

$$
\beta_{A}(x) \leq \max \left\{\beta_{A}(x * y), \beta_{A}(y)\right\} \leq s .
$$

Thus $x \in L\left(\beta_{A} ; s\right)$, and therefore $L\left(\beta_{A} ; s\right)$ is an ideal of $X$. Conversely, assume that for each $t, s \in[0,1]$, the sets $U\left(\alpha_{A} ; t\right)$ and $L\left(\beta_{A} ; s\right)$ are either empty or ideals of $X$. For any $x \in X$, let $\alpha_{A}(x)=t$ and $\beta_{A}(x)=s$. Then $x \in U\left(\alpha_{A} ; t\right) \cap L\left(\beta_{A} ; s\right)$, and so $U\left(\alpha_{A} ; t\right) \neq \varnothing \neq L\left(\beta_{A} ; s\right)$. Since $U\left(\alpha_{A} ; t\right)$ and $L\left(\beta_{A} ; s\right)$ are ideals of $X$, therefore $0 \in$ $U\left(\alpha_{A} ; t\right) \cap L\left(\beta_{A} ; s\right)$. Hence $\alpha_{A}(0) \geq t=\alpha_{A}(x)$ and $\beta_{A}(0) \leq s=\beta_{A}(x)$ for all $x \in X$. If there exist $x^{\prime}, y^{\prime} \in X$ such that $\alpha_{A}\left(x^{\prime}\right)<\min \left\{\alpha_{A}\left(x^{\prime} * y^{\prime}\right), \alpha_{A}\left(y^{\prime}\right)\right\}$, then by taking

$$
t_{0}=\frac{1}{2}\left(\alpha_{A}\left(x^{\prime}\right)+\min \left\{\alpha_{A}\left(x^{\prime} * y^{\prime}\right), \alpha_{A}\left(y^{\prime}\right)\right\}\right),
$$

we have

$$
\alpha_{A}\left(x^{\prime}\right)<t_{0}<\min \left\{\alpha_{A}\left(x^{\prime} * y^{\prime}\right), \alpha_{A}\left(y^{\prime}\right)\right\} .
$$

Hence $x^{\prime} \notin U\left(\alpha_{A} ; t_{0}\right), x^{\prime} * y^{\prime} \in U\left(\alpha_{A} ; t_{0}\right)$ and $y^{\prime} \in\left(\alpha_{A} ; t_{0}\right)$, that is, $U\left(\alpha_{A} ; t_{0}\right)$ is not an ideal of $X$, which is a contradiction. Finally, assume that there exist $a, b \in X$ such that

$$
\beta_{A}(a)>\max \left\{\beta_{A}(a * b), \beta_{A}(b)\right\} .
$$

Taking $s_{0}:=(1 / 2)\left(\beta_{A}(a)+\max \left\{\beta_{A}(a * b), \beta_{A}(b)\right\}\right)$, then

$$
\max \left\{\beta_{A}(a * b), \beta_{A}(b)\right\}<s_{0}<\beta_{A}(a) .
$$

Therefore $a * b \in L\left(\beta_{A} ; s_{0}\right)$ and $b \in L\left(\beta_{A} ; s_{0}\right)$, but $a \notin L\left(\beta_{A} ; s_{0}\right)$, which is a contradiction, this completes the proof.

Let $\Lambda$ be a nonempty subset of $[0,1]$.

THEOREM 3.14. Let $\left\{I_{t} \mid t \in \Lambda\right\}$ be a collection of ideals of $X$ such that

(i) $X=\cup_{t \in \Lambda} I_{t}$,

(ii) $s>t$ if and only if $I_{s} \subset I_{t}$ for all $s, t \in \Lambda$.

Then an IFS $A=\left(\alpha_{A}, \beta_{A}\right)$ in $X$ defined by

$$
\alpha_{A}(x):=\sup \left\{t \in \Lambda \mid x \in I_{t}\right\}, \quad \beta_{A}(x):=\inf \left\{t \in \Lambda \mid x \in I_{t}\right\}
$$

for all $x \in X$ is an intuitionistic fuzzy ideal of $X$.

Proof. According to Theorem 3.13, it is sufficient to show that $U\left(\alpha_{A} ; t\right)$ and $L\left(\beta_{A} ; s\right)$ are ideals of $X$ for every $t \in\left[0, \alpha_{A}(0)\right]$ and $s \in\left[\beta_{A}(0), 1\right]$. In order to prove 
that $U\left(\alpha_{A} ; t\right)$ is an ideal of $X$, we divide the proof into the following two cases:

(i) $t=\sup \{q \in \Lambda \mid q<t\}$,

(ii) $t \neq \sup \{q \in \Lambda \mid q<t\}$.

Case (i) implies that

$$
x \in U\left(\alpha_{A} ; t\right) \Longleftrightarrow x \in I_{q} \quad \forall q<t \Longleftrightarrow x \in \cap_{q<t} I_{q},
$$

so that $U\left(\alpha_{A} ; t\right)=\cap_{q<t} I_{q}$, which is an ideal of $X$. For the case (ii), we claim that $U\left(\alpha_{A} ; t\right)=\cup_{q \geq t} I_{q}$. If $x \in \cup_{q \geq t} I_{q}$, then $x \in I_{q}$ for some $q \geq t$. It follows that $\alpha_{A}(x) \geq$ $q \geq t$, so that $x \in U\left(\alpha_{A} ; t\right)$. This shows that $\cup_{q \geq t} I_{q} \subseteq U\left(\alpha_{A} ; t\right)$. Now assume that $x \notin \cup_{q \geq t} I_{q}$. Then $x \notin I_{q}$ for all $q \geq t$. Since $t \neq \sup \{q \in \Lambda \mid q<t\}$, there exists $\varepsilon>0$ such that $(t-\varepsilon, t) \cap \Lambda=\varnothing$. Hence $x \notin I_{q}$ for all $q>t-\varepsilon$, which means that if $x \in I_{q}$, then $q \leq t-\varepsilon$. Thus $\alpha_{A}(x) \leq t-\varepsilon<t$, and so $x \notin U\left(\alpha_{A} ; t\right)$. Therefore $U\left(\alpha_{A} ; t\right) \subseteq \cup_{q \geq t} I_{q}$, and thus $U\left(\alpha_{A} ; t\right)=\cup_{q \geq t} I_{q}$ which is an ideal of $X$. Next we prove that $L\left(\beta_{A} ; s\right)$ is an ideal of $X$. We consider the following two cases:

(iii) $s=\inf \{r \in \Lambda \mid s<r\}$,

(iv) $s \neq \inf \{r \in \Lambda \mid s<r\}$.

For the case (iii), we have

$$
x \in L\left(\beta_{A} ; s\right) \Longleftrightarrow x \in I_{r} \quad \forall s<r \Longleftrightarrow x \in \cap_{s<r} I_{r},
$$

and hence $L\left(\beta_{A} ; s\right)=\cap_{s<r} I_{r}$ which is an ideal of $X$. For the case (iv) there exists $\varepsilon>0$ such that $(s, s+\varepsilon) \cap \Lambda=\varnothing$. We will show that $L\left(\beta_{A} ; s\right)=\cup_{s \geq r} I_{r}$. If $x \in \cup_{s \geq r} I_{r}$, then $x \in I_{r}$ for some $r \leq s$. It follows that $\beta_{A}(x) \leq r \leq s$ so that $x \in L\left(\beta_{A} ; s\right)$. Hence $\cup_{s \geq r} I_{r} \subseteq L\left(\beta_{A} ; s\right)$. Conversely, if $x \notin \cup_{s \geq r} I_{r}$, then $x \notin I_{r}$ for all $r \leq s$, which implies that $x \notin I_{r}$ for all $r<s+\varepsilon$, that is, if $x \in I_{r}$, then $r \geq s+\varepsilon$. Thus $\beta_{A}(x) \geq s+\varepsilon>s$, that is, $x \notin L\left(\beta_{A} ; s\right)$. Therefore $L\left(\beta_{A} ; s\right) \subseteq \cup_{s \geq r} I_{r}$ and consequently $L\left(\beta_{A} ; s\right)=\cup_{s \geq r} I_{r}$ which is an ideal of $X$. This completes the proof.

A mapping $f: X \rightarrow Y$ of BCK-algebras is called a homomorphism if $f(x * y)=$ $f(x) * f(y)$ for all $x, y \in X$. Note that if $f: X \rightarrow Y$ is a homomorphism of BCKalgebras, then $f(0)=0$. Let $f: X \rightarrow Y$ be a homomorphism of BCK-algebras. For any $\operatorname{IFS} A=\left(\alpha_{A}, \beta_{A}\right)$ in $Y$, we define a new IFS $A^{f}=\left(\alpha_{A}^{f}, \beta_{A}^{f}\right)$ in $X$ by

$$
\alpha_{A}^{f}(x):=\alpha_{A}(f(x)), \quad \beta_{A}^{f}(x):=\beta_{A}(f(x)) \quad \forall x \in X .
$$

THEOREM 3.15. Let $f: X \rightarrow Y$ be a homomorphism of BCK-algebras. If an IFS $A=$ $\left(\alpha_{A}, \beta_{A}\right)$ in $Y$ is an intuitionistic fuzzy ideal of $Y$, then an $\operatorname{IFS} A^{f}=\left(\alpha_{A}^{f}, \beta_{A}^{f}\right)$ in $X$ is an intuitionistic fuzzy ideal of $X$.

Proof. We first have that

$$
\begin{aligned}
& \alpha_{A}^{f}(x)=\alpha_{A}(f(x)) \leq \alpha_{A}(0)=\alpha_{A}(f(0))=\alpha_{A}^{f}(0), \\
& \beta_{A}^{f}(x)=\beta_{A}(f(x)) \geq \beta_{A}(0)=\beta_{A}(f(0))=\beta_{A}^{f}(0)
\end{aligned}
$$

for all $x \in X$. Let $x, y \in X$. Then 


$$
\begin{aligned}
\min \left\{\alpha_{A}^{f}(x * y), \alpha_{A}^{f}(y)\right\} & =\min \left\{\alpha_{A}(f(x * y)), \alpha_{A}(f(y))\right\} \\
& =\min \left\{\alpha_{A}(f(x) * f(y)), \alpha_{A}(f(y))\right\} \\
& \leq \alpha_{A}(f(x))=\alpha_{A}^{f}(x), \\
\max \left\{\beta_{A}^{f}(x * y), \beta_{A}^{f}(y)\right\} & =\max \left\{\beta_{A}(f(x * y)), \beta_{A}(f(y))\right\} \\
& =\max \left\{\beta_{A}(f(x) * f(y)), \beta_{A}(f(y))\right\} \\
& \geq \beta_{A}(f(x))=\beta_{A}^{f}(x) .
\end{aligned}
$$

Hence $A^{f}=\left(\alpha_{A}^{f}, \beta_{A}^{f}\right)$ is an intuitionistic fuzzy ideal of $X$.

If we strengthen the condition of $f$, then we can construct the converse of Theorem 3.15 as follows.

THEOREM 3.16. Let $f: X \rightarrow Y$ be an epimorphism of BCK-algebras and let $A=$ $\left(\alpha_{A}, \beta_{A}\right)$ be an IFS in $Y$. If $A^{f}=\left(\alpha_{A}^{f}, \beta_{A}^{f}\right)$ is an intuitionistic fuzzy ideal of $X$, then $A=\left(\alpha_{A}, \beta_{A}\right)$ is an intuitionistic fuzzy ideal of $Y$.

Proof. For any $x \in Y$, there exists $a \in X$ such that $f(a)=x$. Then

$$
\begin{aligned}
& \alpha_{A}(x)=\alpha_{A}(f(a))=\alpha_{A}^{f}(a) \leq \alpha_{A}^{f}(0)=\alpha_{A}(f(0))=\alpha_{A}(0), \\
& \beta_{A}(x)=\beta_{A}(f(a))=\beta_{A}^{f}(a) \geq \beta_{A}^{f}(0)=\beta_{A}(f(0))=\beta_{A}(0) .
\end{aligned}
$$

Let $x, y \in Y$. Then $f(a)=x$ and $f(b)=y$ for some $a, b \in X$. It follows that

$$
\begin{aligned}
\alpha_{A}(x) & =\alpha_{A}(f(a))=\alpha_{A}^{f}(a) \\
& \geq \min \left\{\alpha_{A}^{f}(a * b), \alpha_{A}^{f}(b)\right\} \\
& =\min \left\{\alpha_{A}(f(a * b)), \alpha_{A}(f(b))\right\} \\
& =\min \left\{\alpha_{A}(f(a) * f(b)), \alpha_{A}(f(b))\right\} \\
& =\min \left\{\alpha_{A}(x * y), \alpha_{A}(y)\right\}, \\
\beta_{A}(x) & =\beta_{A}(f(a))=\beta_{A}^{f}(a) \\
& \leq \max \left\{\beta_{A}^{f}(a * b), \beta_{A}^{f}(b)\right\} \\
& =\max \left\{\beta_{A}(f(a * b)), \beta_{A}(f(b))\right\} \\
& =\max \left\{\beta_{A}(f(a) * f(b)), \beta_{A}(f(b))\right\} \\
& =\max \left\{\beta_{A}(x * y), \beta_{A}(y)\right\} .
\end{aligned}
$$

This completes the proof.

Let $\operatorname{IF}(X)$ be the family of all intuitionistic fuzzy ideals of $X$ and let $t \in[0,1]$. Define binary relations $U^{t}$ and $L^{t}$ on $\operatorname{IF}(X)$ as follows:

$$
(A, B) \in U^{t} \Longleftrightarrow U\left(\alpha_{A} ; t\right)=U\left(\alpha_{B} ; t\right), \quad(A, B) \in L^{t} \Longleftrightarrow L\left(\beta_{A} ; t\right)=L\left(\beta_{B} ; t\right),
$$

respectively, for $A=\left(\alpha_{A}, \beta_{A}\right)$ and $B=\left(\alpha_{B}, \beta_{B}\right)$ in $\operatorname{IF}(X)$. Then clearly $U^{t}$ and $L^{t}$ are 
equivalence relations on $\operatorname{IF}(X)$. For any $A=\left(\alpha_{A}, \beta_{A}\right) \in \operatorname{IF}(X)$, let $[A]_{U^{t}}$ (respectively, $[A]_{L^{t}}$ ) denote the equivalence class of $A$ modulo $U^{t}$ (respectively, $L^{t}$ ), and denote by $\operatorname{IF}(X) / U^{t}$ (respectively, $\operatorname{IF}(X) / L^{t}$ ) the system of all equivalence classes modulo $U^{t}$ (respectively, $L^{t}$ ); so

$$
\operatorname{IF}(X) / U^{t}:=\left\{[A]_{U^{t}} \mid A=\left(\alpha_{A}, \beta_{A}\right) \in \operatorname{IF}(X)\right\},
$$

respectively,

$$
\operatorname{IF}(X) / L^{t}:=\left\{[A]_{L^{t}} \mid A=\left(\alpha_{A}, \beta_{A}\right) \in \operatorname{IF}(X)\right\} .
$$

Now let $I(X)$ denote the family of all ideals of $X$ and let $t \in[0,1]$. Define maps $f_{t}$ and $g_{t}$ from $\operatorname{IF}(X)$ to $I(X) \cup\{\varnothing\}$ by $f_{t}(A)=U\left(\alpha_{A} ; t\right)$ and $g_{t}(A)=L\left(\beta_{A} ; t\right)$, respectively, for all $A=\left(\alpha_{A}, \beta_{A}\right) \in \operatorname{IF}(X)$. Then $f_{t}$ and $g_{t}$ are clearly well defined.

THEOREM 3.17. For any $t \in(0,1)$ the maps $f_{t}$ and $g_{t}$ are surjective from $\operatorname{IF}(X)$ to $I(X) \cup\{\varnothing\}$.

Proof. Let $t \in(0,1)$. Note that $\mathbf{0}_{\sim}=(\mathbf{0}, \mathbf{1})$ is in $\operatorname{IF}(X)$, where $\mathbf{0}$ and $\mathbf{1}$ are fuzzy sets in $X$ defined by $\mathbf{0}(x)=0$ and $\mathbf{1}(x)=1$ for all $x \in X$. Obviously $f_{t}\left(\mathbf{0}_{\sim}\right)=U(\mathbf{0} ; t)=$ $\varnothing=L(\mathbf{1} ; t)=g_{t}\left(\mathbf{0}_{\sim}\right)$. Let $G(\neq \varnothing) \in I(X)$. For $G_{\sim}=\left(\chi_{G}, \bar{X}_{G}\right) \in \operatorname{IF}(X)$, we have $f_{t}\left(G_{\sim}\right)=$ $U\left(\chi_{G} ; t\right)=G$ and $g_{t}\left(G_{\sim}\right)=L\left(\bar{\chi}_{G} ; t\right)=G$. Hence $f_{t}$ and $g_{t}$ are surjective.

THEOREM 3.18. The quotient sets $\operatorname{IF}(X) / U^{t}$ and $\operatorname{IF}(X) / L^{t}$ are equipotent to $I(X) \cup$ $\{\varnothing\}$ for every $t \in(0,1)$.

Proof. For $t \in(0,1)$ let $f_{t}^{*}$ (respectively, $g_{t}^{*}$ ) be a map from $\operatorname{IF}(X) / U^{t}$ (respectively, $\left.\operatorname{IF}(X) / L^{t}\right)$ to $I(X) \cup\{\varnothing\}$ defined by $f_{t}^{*}\left([A]_{U^{t}}\right)=f_{t}(A)$ (respectively, $g_{t}^{*}\left([A]_{L^{t}}\right)=$ $\left.g_{t}(A)\right)$ for all $A=\left(\alpha_{A}, \beta_{A}\right) \in \operatorname{IF}(X)$. If $U\left(\alpha_{A} ; t\right)=U\left(\alpha_{B} ; t\right)$ and $L\left(\beta_{A} ; t\right)=L\left(\beta_{B} ; t\right)$ for $A=\left(\alpha_{A}, \beta_{A}\right)$ and $B=\left(\alpha_{B}, \beta_{B}\right)$ in $\operatorname{IF}(X)$, then $(A, B) \in U^{t}$ and $(A, B) \in L^{t}$; hence $[A]_{U^{t}}=[B]_{U^{t}}$ and $[A]_{L^{t}}=[B]_{L^{t}}$. Therefore the maps $f_{t}^{*}$ and $g_{t}^{*}$ are injective. Now let $G(\neq \varnothing) \in I(X)$. For $G_{\sim}=\left(\chi_{G}, \bar{\chi}_{G}\right) \in \operatorname{IF}(X)$, we have

$$
\begin{gathered}
f_{t}^{*}\left(\left[G_{\sim}\right]_{U^{t}}\right)=f_{t}\left(G_{\sim}\right)=U\left(\chi_{G} ; t\right)=G, \\
g_{t}^{*}\left(\left[G_{\sim}\right]_{L^{t}}\right)=g_{t}\left(G_{\sim}\right)=L\left(\bar{\chi}_{G} ; t\right)=G .
\end{gathered}
$$

Finally, for $\mathbf{0}_{\sim}=(\mathbf{0}, \mathbf{1}) \in \operatorname{IF}(X)$ we get

$$
\begin{gathered}
f_{t}^{*}\left(\left[\mathbf{0}_{\sim}\right]_{U^{t}}\right)=f_{t}\left(\mathbf{0}_{\sim}\right)=U(\mathbf{0} ; t)=\varnothing, \\
g_{t}^{*}\left(\left[\mathbf{0}_{\sim}\right]_{L^{t}}\right)=g_{t}\left(\mathbf{0}_{\sim}\right)=L(\mathbf{0} ; t)=\varnothing .
\end{gathered}
$$

This shows that $f_{t}^{*}$ and $g_{t}^{*}$ are surjective. This completes the proof.

For any $t \in[0,1]$, we define another relation $R^{t} \operatorname{on} \operatorname{IF}(X)$ as follows:

$$
(A, B) \in R^{t} \Longleftrightarrow U\left(\alpha_{A} ; t\right) \cap L\left(\beta_{A} ; t\right)=U\left(\alpha_{B} ; t\right) \cap L\left(\beta_{B} ; t\right)
$$


for any $A=\left(\alpha_{A}, \beta_{A}\right), B=\left(\alpha_{B}, \beta_{B}\right) \in \operatorname{IF}(X)$. Then the relation $R^{t}$ is also an equivalence relation on $\operatorname{IF}(X)$.

THEOREM 3.19. For any $t \in(0,1)$, the map $\phi_{t}: \operatorname{IF}(X) \rightarrow I(X) \cup\{\varnothing\}$ defined by $\phi_{t}(A)=f_{t}(A) \cap g_{t}(A)$ for each $A=\left(\alpha_{A}, \beta_{A}\right) \in \operatorname{IF}(X)$ is surjective.

Proof. Let $t \in(0,1)$. For $\mathbf{0}_{\sim}=(\mathbf{0}, \mathbf{1}) \in \operatorname{IF}(X)$,

$$
\phi_{t}\left(\mathbf{0}_{\sim}\right)=f_{t}\left(\mathbf{0}_{\sim}\right) \cap g_{t}\left(\mathbf{0}_{\sim}\right)=U(\mathbf{0} ; t) \cap L(\mathbf{1} ; t)=\varnothing .
$$

For any $H \in \operatorname{IF}(X)$, there exists $H_{\sim}=\left(\chi_{H}, \bar{\chi}_{H}\right) \in \operatorname{IF}(X)$ such that

$$
\phi_{t}\left(H_{\sim}\right)=f_{t}\left(H_{\sim}\right) \cap g_{t}\left(H_{\sim}\right)=U\left(\chi_{H} ; t\right) \cap L\left(\bar{x}_{H} ; t\right)=H .
$$

This completes the proof.

THEOREM 3.20. For any $t \in(0,1)$, the quotient set $\operatorname{IF}(X) / R^{t}$ is equipotent to $I(X) \cup\{\varnothing\}$.

Proof. Let $t \in(0,1)$ and let $\phi_{t}^{*}: \operatorname{IF}(X) / R^{t} \rightarrow I(X) \cup\{\varnothing\}$ be a map defined by $\phi_{t}^{*}\left([A]_{R^{t}}\right)=\phi_{t}(A)$ for all $[A]_{R^{t}} \in \operatorname{IF}(X) / R^{t}$. If $\phi_{t}^{*}\left([A]_{R^{t}}\right)=\phi_{t}^{*}\left([B]_{R^{t}}\right)$ for any $[A]_{R^{t}}$, $[B]_{R^{t}} \in \operatorname{IF}(X) / R^{t}$, then $f_{t}(A) \cap g_{t}(A)=f_{t}(B) \cap g_{t}(B)$, that is, $U\left(\alpha_{A} ; t\right) \cap L\left(\beta_{A} ; t\right)=$ $U\left(\alpha_{B} ; t\right) \cap L\left(\beta_{B} ; t\right)$, hence $(A, B) \in R^{t}$. It follows that $[A]_{R^{t}}=[B]_{R^{t}}$ so that $\phi_{t}^{*}$ is injective. For $\mathbf{0}_{\sim}=(\mathbf{0}, \mathbf{1}) \in \operatorname{IF}(X)$,

$$
\phi_{t}^{*}\left(\left[\mathbf{0}_{\sim}\right]_{R^{t}}\right)=\phi_{t}\left(\mathbf{0}_{\sim}\right)=f_{t}\left(\mathbf{0}_{\sim}\right) \cap g_{t}\left(\mathbf{0}_{\sim}\right)=U(\mathbf{0} ; t) \cap L(\mathbf{1} ; t)=\varnothing .
$$

If $H \in \operatorname{IF}(X)$, then for $H_{\sim}=\left(\chi_{H}, \bar{\chi}_{H}\right) \in \operatorname{IF}(X)$, we have

$$
\phi_{t}^{*}\left(\left[H_{\sim}\right]_{R^{t}}\right)=\phi\left(H_{\sim}\right)=f_{t}\left(H_{\sim}\right) \cap g_{t}\left(H_{\sim}\right)=U\left(\chi_{H} ; t\right) \cap L\left(\bar{x}_{H} ; t\right)=H .
$$

Hence $\phi_{t}^{*}$ is surjective, this completes the proof.

ACKNOWLEDGEMENT. The first author was supported by Korea Research Foundation Grant (KRF-99-005-D00003).

\section{REFERENCES}

[1] K. T. Atanassov, Intuitionistic fuzzy sets, Fuzzy Sets and Systems 20 (1986), no. 1, 87-96. MR 87f:03151. Zbl 631.03040.

[2] _ New operations defined over the intuitionistic fuzzy sets, Fuzzy Sets and Systems 61 (1994), no. 2, 137-142. CMP 1262 464. Zbl 824.04004.

[3] Y. B. Jun, A note on fuzzy ideals in BCK-algebras, Math. Japon. 42 (1995), no. 2, 333-335. CMP 1356 395. Zbl 834.06018.

[4] _ Finite valued fuzzy ideals in BCK-algebras, J. Fuzzy Math. 5 (1997), no. 1, 111-114. CMP 1441 020. Zbl 868.06010.

[5] _ Characterizations of Noetherian BCK-algebras via fuzzy ideals, Fuzzy Sets and Systems 108 (1999), no. 2, 231-234. CMP 1720 432. Zbl 940.06014.

[6] Y. B. Jun, S. M. Hong, S. J. Kim, and S. Z. Song, Fuzzy ideals and fuzzy subalgebras of BCKalgebras, J. Fuzzy Math. 7 (1999), no. 2, 411-418. MR 2000c:06040. Zbl 943.06010.

[7] Y. B. Jun and E. H. Roh, Fuzzy commutative ideals of BCK-algebras, Fuzzy Sets and Systems 64 (1994), no. 3, 401-405. MR 95e:06051. Zbl 846.06011. 
[8] J. Meng, Y. B. Jun, and H. S. Kim, Fuzzy implicative ideals of BCK-algebras, Fuzzy Sets and Systems 89 (1997), no. 2, 243-248. MR 98a:06033. Zbl 914.06009.

[9] L. A. Zadeh, Fuzzy sets, Information and Control 8 (1965), 338-353. MR 36\#2509. Zbl 139.24606.

Young Bae Jun: Department of MAthematics Education, Gyeongsang National UniVERSITY, CHINJU 660-701, KOREA

E-mail address: ybjun@nongae.gsnu.ac. kr

KyUng Ho Kim: Department of Mathematics, ChunguU National University, ChungJu 380-702, KOREA

E-mail address: ghkim@gukwon.chungju.ac.kr 


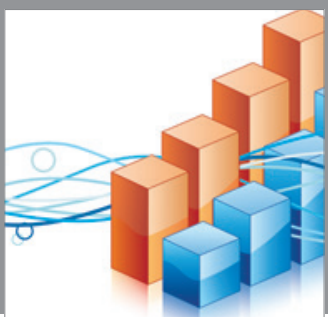

Advances in

Operations Research

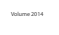

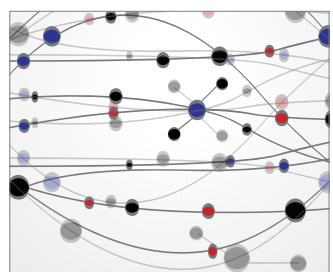

\section{The Scientific} World Journal
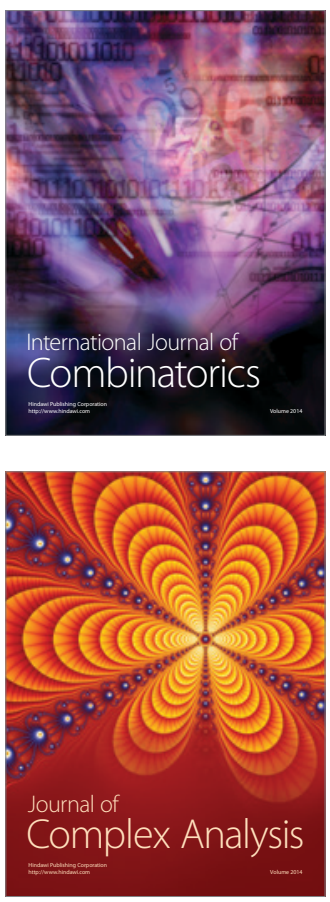

International Journal of

Mathematics and

Mathematical

Sciences
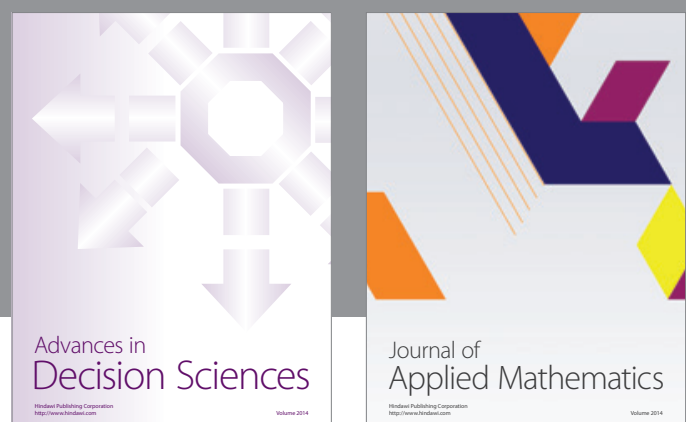

Journal of

Applied Mathematics
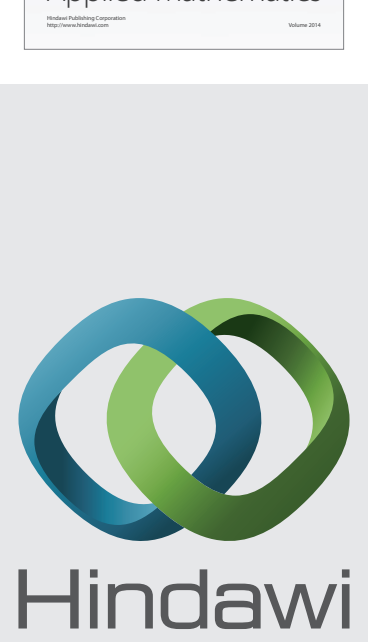

Submit your manuscripts at http://www.hindawi.com
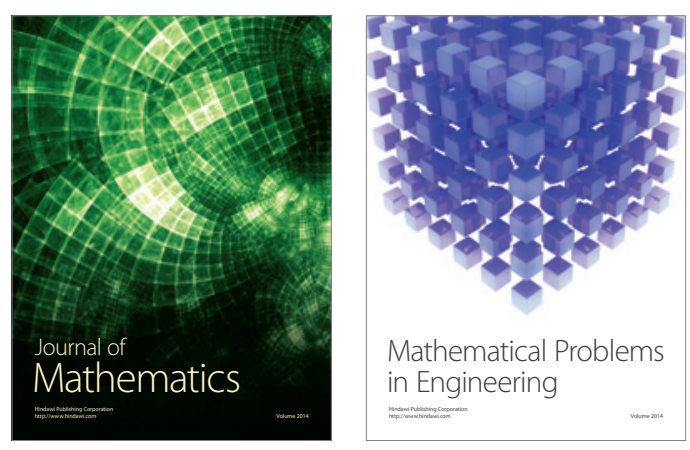

Mathematical Problems in Engineering
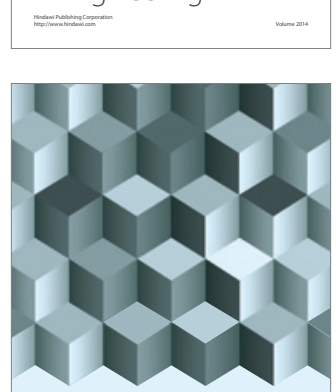

Journal of

Function Spaces
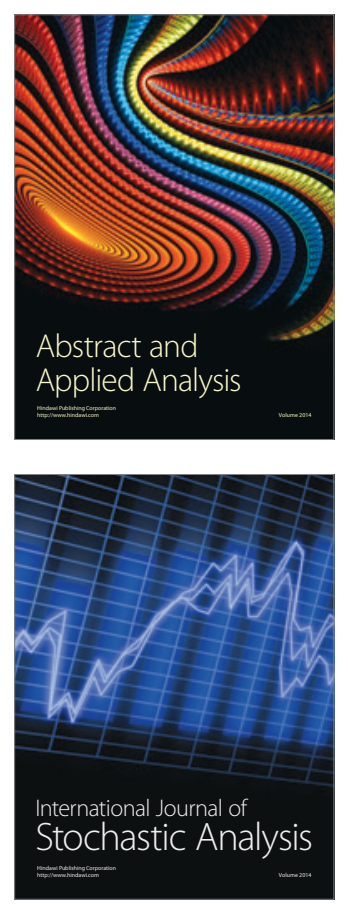

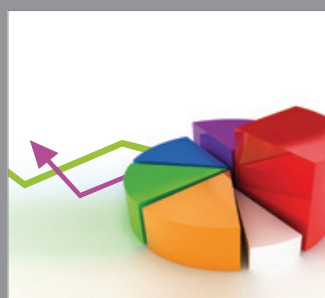

ournal of

Probability and Statistics

Promensencen
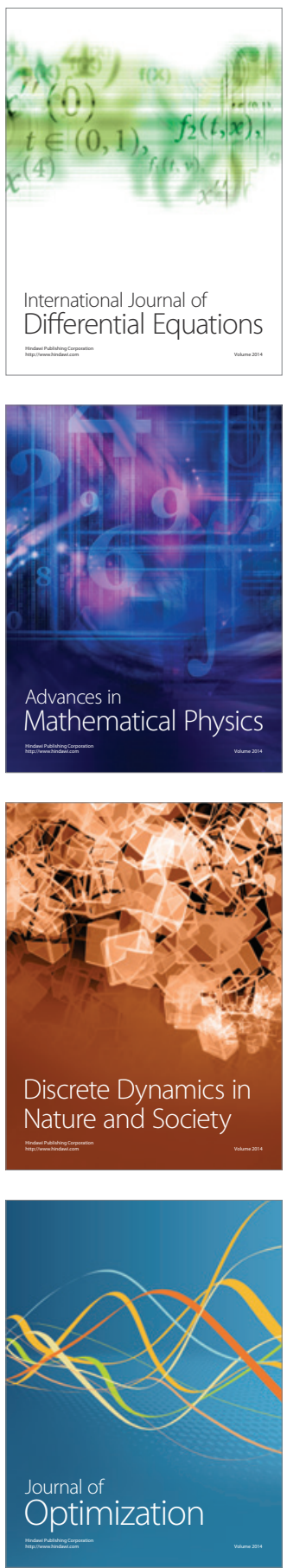